\title{
B-Spline Curve Fitting and the Feature Point Extraction of Fiber Image
}

\author{
Xiao-chun Chen ${ }^{1, \mathrm{a}}$, Xiong $\mathrm{Li}^{1,2}$ \\ ${ }^{1}$ Key Laborary of EDA, Research Institute of Tsinghua University in Shenzhen, Shenzhen, China; \\ ${ }^{2}$ College of Information Engineering, Yunnan University, Kunming, China; \\ aemail:chenxc@tsinghua-sz.org,
}

Keywords: B-spline; Complex field nonlinear anisotropic diffusion process; fiber image

\begin{abstract}
Fiber images taken by optical microscope tend to have poor focus, uneven brightness or blurred edge. Using mathematical morphology method, such as open or close operation to preprocess the fiber image, which is likely to lead to distortion of the edge of the fiber image and affect the subsequent separation operation. B-spline curve is used to fit the background of the image, then the fitting background is subtracted from the original image. Next, the complex domain nonlinear anisotropic diffusion process is used to denoise and enhance the image edge. Finally, Binarized the image and filling area to get a clear picture of the target fiber image.
\end{abstract}

\section{Introduction}

At present, the method of computer automatic recognition technology of fiber image has achieved more attention by domestic and foreign researchers[1-4], several features for evaluation have also been put forward. However, the available technology of intelligent fiber identification depends on a number of extraction of feature indicators, such as longitudinal and cross-sectional morphological characteristics of cotton fibers. These indicators are often based on precise measurements of the individual fibers, but the actual test of the required sample image usually includes a plurality of fibers. So how to separate a plurality of overlapping target is the first problem to solve.

Fiber images taken by optical microscope tends to have poor focus, uneven brightness or blurred edge. Using mathematical morphology method, such as open or close operation to preprocess the fiber image, which is likely to lead to distortion of the edge of the fiber image and affect the subsequent separation operation. Medical image processing[5] can adopt methods as image contour pits, watershed algorithm, and morphology and corrosion algorithm for segmentation of overlapping cells or drugs. Their shapes are relatively regular but different from those of the fibers. For a fiber image, the phenomenon of cross, torsion or adhesion between the fibers makes the separation very difficult.

In this paper, the B-spline curve fitting and complex domain nonlinear anisotropic diffusion process are used to deal with the edge of fiber image. B-spline curve is used to fit the background of the image, and the fitting background is subtracted from the original image. Next, the complex domain nonlinear anisotropic diffusion process is used to denoise and enhance the image edge. Finally, the binarization and region filling processing is used to get the clear fiber object.

\section{Algorithm Description}

\section{The B-spline curve gray fitting of fiber image}

The B-spline curve fitting method is used to get the fitting background of image, then the fitting background is subtracted from the original fiber image to obtain the target image. This process is able to remove the image background and improve the uneven illumination impact of image. The algorithm uses a bi-cubic B-spline curve to fit the background, restricted by the least squares.

The gray value of fiber image is defined as $F\left(x_{i}, x_{j}\right)$, where $1<\mathrm{i}<\mathrm{M}, 1<\mathrm{j}<\mathrm{N}$. Assuming that there exist $(m+1) \times(n+1)$ pixel points in an image, where $m>M, n>N$. For a given range: $X_{u}: 0=$ $\mathrm{u}_{0}<\ldots<\mathrm{u}_{\mathrm{m}}=1, \mathrm{Y}_{\mathrm{v}}: 0=\mathrm{v}_{0}<\ldots<\mathrm{v}_{\mathrm{n}}=1$, the $\mathrm{X}_{\mathrm{u}}$ and $\mathrm{Y}_{\mathrm{v}}$ respectively have $(\mathrm{m}+1)$ and $(\mathrm{n}+1)$ cubic B-spline function which are recorded as $B_{i}(u)$ and $B_{j}(v)$, where $i=0,1, \ldots m$, and $j=0,1, \ldots n$. 
Accordingly, the background of the image can be expressed as follows:

$$
G(u, v)=\sum_{i=0}^{m} \sum_{j=0}^{n} B_{i}(u) B_{j}(v) P_{i, j}
$$

Where: $\mathrm{P}_{\mathrm{i}, \mathrm{j}}$ as a pixel point, $\mathrm{B}_{\mathrm{i}}(\mathrm{u})$ or $\mathrm{B}_{\mathrm{j}}(\mathrm{u})$ is the cubic $\mathrm{B}$-spline base function, they can also be expressed as formula (2):

$$
\mathrm{B}_{\mathrm{i}}(u)= \begin{cases}0 ; & \mathrm{i}<\mathrm{k} \text { or } \mathrm{i}>(\mathrm{k}+3) \\ 1 / 6\left(-\mathrm{u}^{3}+3 \mathrm{u}^{2}-3 \mathrm{u}+1\right) ; & \mathrm{i}=\mathrm{k} \\ 1 / 6\left(3 \mathrm{u}^{3}-6 \mathrm{u}^{3}+4\right) ; & \mathrm{i}=\mathrm{k} \\ 1 / 6\left(-3 \mathrm{u}^{3}+3 \mathrm{u}^{3}+3 \mathrm{u}+1\right) ; & \mathrm{i}=\mathrm{k}+2 \\ 1 / 6 \mathrm{u}^{3} ; & \mathrm{i}=\mathrm{k}\end{cases}
$$

where: $\mathrm{k}=0,1, \ldots \mathrm{m}-1$.

The curve fitting is not completely dependent on the gray value of a given fiber image. In order to control the fitting precision, the formula (1) is applied here to calculate the pixel points $\mathrm{P}_{\mathrm{i}, \mathrm{j}}$ conversely. According to the criterion of least squares, the objective function $\delta$ is defined as formula (3).

$$
\delta=\sum_{i=0}^{m} \sum_{j=0}^{n}\left|\mathrm{~F}\left(x_{i}, y_{j}\right)-G\left(u_{i}, v_{j}\right)\right|
$$

An equation group includes $(m+1) \times(n+1)$ equations is obtained by derivation and set to zero for pixel point $\mathrm{P}_{\mathrm{i}, \mathrm{j}}$ such as $\partial \delta / \partial \mathrm{P}_{\mathrm{i}, j}=0$. By solving these equations, the least squares fitting curve $\mathrm{G}$ $(\mathrm{U}, \mathrm{V})$ is obtained, which is the background of the fiber image.

\section{Nonlinear anisotropic diffusion process in complex domain}

A directional distribution coefficient is used in the process of complex domain nonlinear anisotropic diffusion filter to replace the Gauss Convolution method. This method can get good results for preserving image details and denoise [6,7]. The size of fiber image I is defined as $\mathrm{M} \times \mathrm{N}$, and the processing is descripted as follows:

1. According to the equation (4), the image gradients in all directions is calculated as follows:

$$
\begin{aligned}
& \text { jn }=[I(1,:) ; I(1: M-1,:)]-I ; \\
& j s=[I(2: M,:) ; I(M,:)]-I ; \\
& j e=[I(:, 2: N) ; I(:, N)]-I ; \\
& j w=[I(:, 1) ; I(:, 1: N-1]-I ;
\end{aligned}
$$

2. According to the formula (5), the diffusion coefficient of the image in all directions is calculated as follows:

$$
\mathrm{C}(j)=\frac{e^{i \theta}}{1+\left(\frac{\operatorname{Im}(j)}{k \theta}\right)^{2}}
$$

For the formula (5), $\mathrm{j}$ is the gradient of the image in all directions, the phase angle $\theta$ is very small $(\theta<<1), k$ is the edge threshold. when $j=j n$, js, je, jw, the corresponding diffusion coefficients are $\mathrm{Cn}, \mathrm{Cs}, \mathrm{Ce}, \mathrm{Cw}$.

3. The object image is obtained from the formula (6) based on the complex domain nonlinear anisotropic diffusion process:

$$
\mathrm{I}=I+d t^{*}\left(C n .{ }^{*} j n+C s . * j s+C e . * j e+C w . * j w\right)
$$




\section{Feature extraction}

There are some extensive researches on the feature point(also called the inflection point) extraction of image in domestic and foreign[8,9]. The feature point can be obtained by using the Equation (7) to calculate the curvature of each pixel to get the change of curvature. On the other hand, the feature point often contains some convex hulls called "pseudo feature point" due to the noise which are produced in the image pick up or preprocessing. On this situation, a good elimination result can be achieved by combination of Gaussian filter with "Ellipse support area" [10].

$$
\mathrm{k}_{\mathrm{i}}=\frac{\Delta x_{i} \Delta^{2} y_{i}-\Delta^{2} x_{i} \Delta y_{i}}{\left[\left(\Delta x_{i}\right)^{2}+\left(\Delta y_{i}\right)^{2}\right]^{3 / 2}}, i=1,2, \ldots, N
$$

where:

$\Delta \mathrm{x}_{i}=\left(x_{i+1}-x_{i-1}\right) / 2 ; \Delta y_{i}=\left(y_{i+1}-y_{i-1}\right) / 2 ; \Delta^{2} \mathrm{x}_{i}=\left(x_{i+1}-x_{i-1}\right) / 2 ; \Delta^{2} \mathrm{y}_{i}=\left(y_{i+1}-y_{i-1}\right) / 2 ; \mathrm{N}$ is the number of pixels of the image profile.

For a given elliptic function $\mathrm{f}(\mathrm{x})$, a vertex of the long axis that is the ellipse pixel $\mathrm{Pi}$, then multiple points are selected to form support areas on both ends of the point of the profile curve. The formula (8) is used here to calculate the curvature of each point on the ellipse to obtain the maximum or minimum curvature of the vertex, and determine the mean curvature of the contour. The mean curvature and experience coefficient are used to get a threshold T_curve, then the bump "pseudo inflection point" can be eliminated, and the true feature point of the fiber image can be obtained.

$$
k(x)=\left|\frac{f^{\prime}(x)}{\left[1+f^{\prime}(x)\right]}\right|=\frac{a^{4} b}{\left[(b x)^{2}-(a x)^{2}+a^{4}\right]^{3 / 2}}
$$

where:

$f(x)=\left[b^{2}-(b x / a)^{2}\right]^{1 / 2}$. a and $b$ corresponding to the minor and major axes of the ellipse, $x \in[-a$, a].

\section{Results and Discussion}

\section{Image processing}

The optical microscope image for two cotton fibers is shown in Figure 1(a). In order to facilitate subsequent processing steps such as the image segmentation or other deep treatment, the following steps is used here:

(1) B-spline curve is used to fit the background of the image, and the fitting background is subtracted from the original image to get the Figure 1(b). This step can remove image background and solve the problem of uneven illumination.

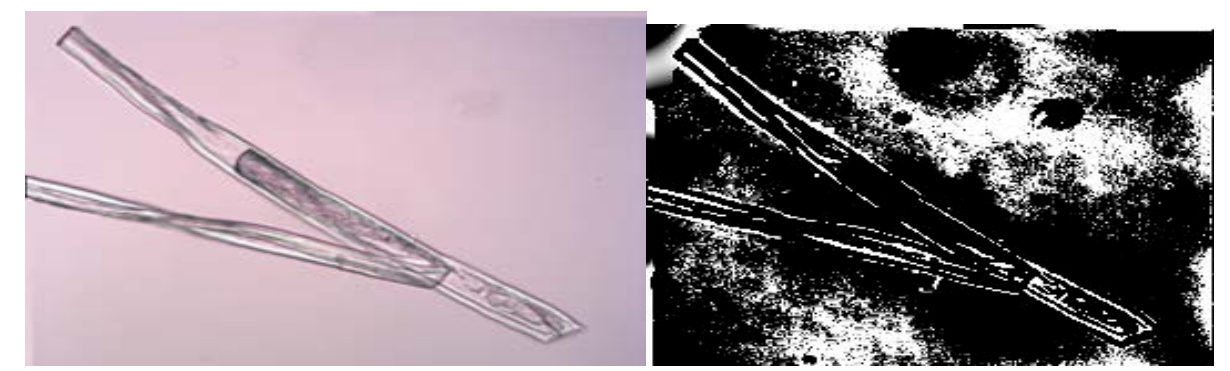

(a) Original image

(b) B-spline curve fitting 

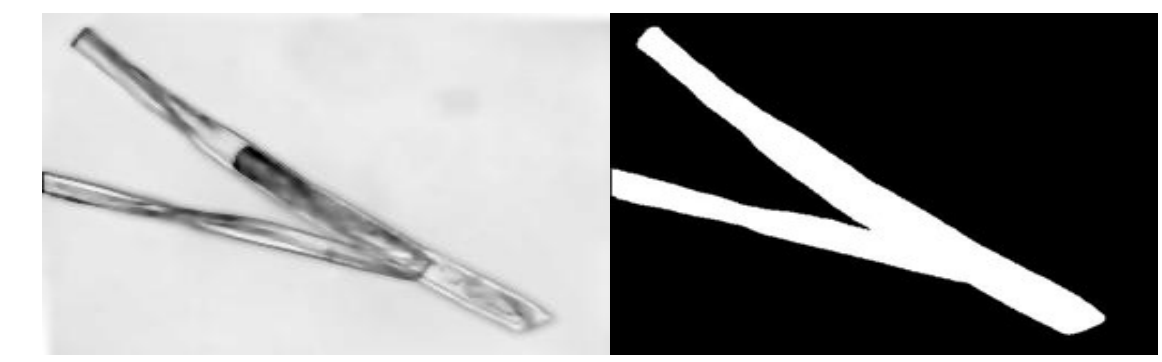

$\begin{array}{ll}\text { (c) Complex domain filter } & \text { (d) Target fiber image }\end{array}$

Fig.1. Based on B-spline curve fitting pre-processing image

(2) There exists some serious noise in the image b, the complex domain nonlinear anisotropic diffusion process is used to filter noise and enhance the object edge.

(3) The binarization and region filling processing are applied to get the clear fiber object d.

\section{Comparison of two methods}

Two processing methods are used to deal with the fiber image shown in figure 2(a). For method 1, the mathematical morphology process, such as open or close operation, is used here, then figure 2(b) is obtained. The deficiency of this method is that the image's cross and adhesion are prone to distortion, some of the smaller angle inflection point is often passive off.

For method 2, B-spline curve fitting is used here. Under the help of the complex domain nonlinear anisotropic diffusion filter, the inflection point in the position of fiber connection or adhesion is clearer than that of figure 2(b), the distortion level is significantly reduced.

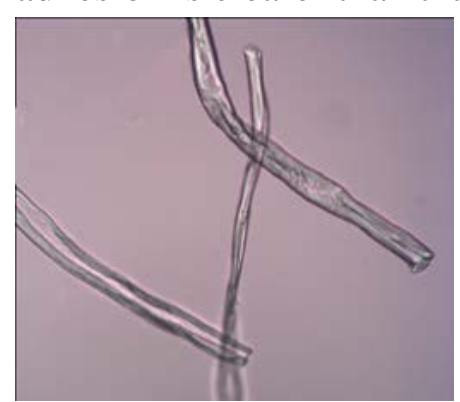

(a)Original image

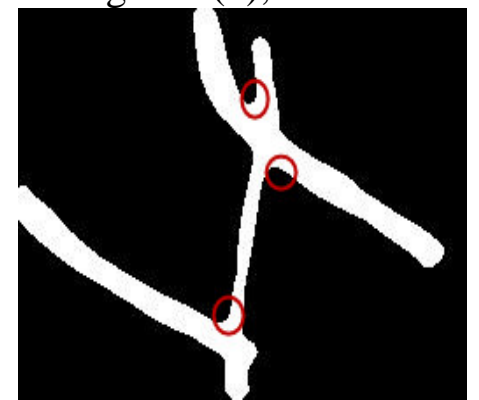

(b) Method 1 result

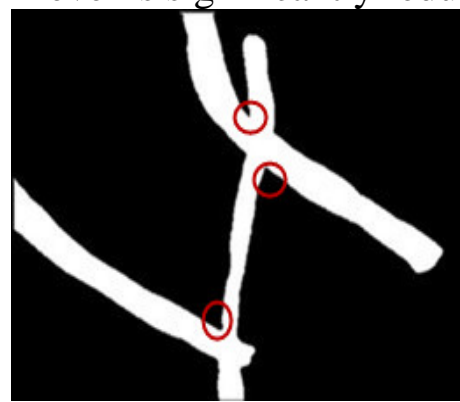

(c) Method 2 result

Fig.2. Comparison of the two method image processing

\section{Extraction of the feature points}

The method described in the feature extraction section of this paper can be realized by programming and applied for figure 1(d) and figure 2(c) respectively, consequently all the feature point can be extracted, shown in figure 3 .

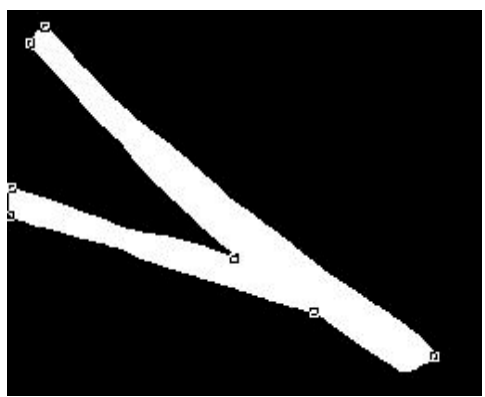

(a)

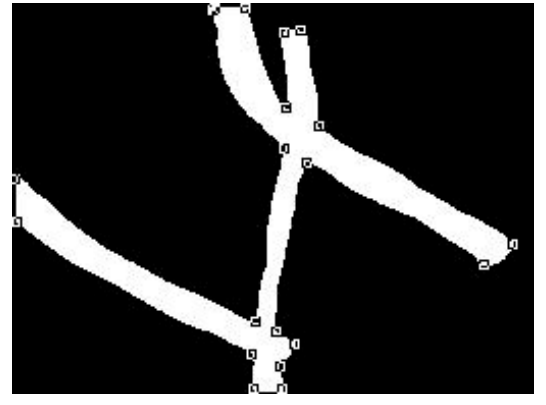

(b)

Fig.3. Feature extraction

The inflection points in a image are important feature points for the identification of fiber image. The extraction method of these inflection points can be referred to the formula (7) and (8). Using the important information provided by these feature points, the adhesion points of the fiber 
image can be found, which provides a basis for subsequent image separation[11].

\section{Conclusion}

The question of the edge processing of fiber image is focused in this paper. The B- spline curve fitting and complex domain nonlinear anisotropic diffusion process have also been applied to deal with the edge of fiber image. The experiment result shows that the method can reduce the background noise and preserve the details of the position of the image adhesion, which is very good for the subsequent image feature points extraction. The image including 2 or 3 overlapped or adhesion fiber as examples has also been applied to validate the ability of algorithm. The result shows that the method can get the concise and clear image edge, and extract the important feature points of the image.

\section{Acknowledgement}

In this paper, the research was sponsored by The Integration Project of Production Teaching and Research by Guangdong Province and Ministry of Education(2012B091100495) and Special fund for the development of strategic emerging industries of Shenzhen (JCYJ20130401172054839).

\section{References}

[1] Zhang chuanxiong, Wu xiongying. The Application of Image Processing Techonology to the Characteristic Parameters of Cellulose Fibers. Journal of Donghua University. 2006, 32(1):46-49.

[2] Huang. Y, Xu. B. Image analysis for cotton fibers, Part I :longitudinal measurements. Textile Research Journal, 2002, 72( 8) : 713-720.

[3] Ying Lebin, Dai Liankui. Single Fiber identification of cotton/flax fabric based on longitudinal view of microscopic fiber images. Journal of Textile Research. 2012, 33(4): 12-18.

[4] Ren Shuaijie, Zhang Wensheng. Measuring Diameter and curvature of fibers Based on Image Analysis. Journal of Image and Graphics. 2008, 13(6): 1153-1158.

[5] Fu Rong, Shen Hong, Chen Hao. Research of Automatically Separating Algorithm for Overlap Cell Based on Searching Concave Spot. Computer Engineering and Applications. 2007, 43(17): 21-23, 28.

[6] Perona P, M al ik J. Scale space and edge detect ion u sing an isotropic d iffusion[J]. IEEE Transactions on Pattern Analysis and Machine Intelligence, 1990, 12(7):629-639.

[7] QIAN Wei-xin,LIU Rui-gen. The Anisotropic Diffusion Methods Based on the Directions of the Image Feature[J]. Journal of Image and Graphics, 2006, 11(6):818-822.

[8] Li H, Liu W Y, Zhu Y T, et al. A uniform model of corner detection based on morphology [J].Computer Engineering and Applications. 2002,7(6): 543-547.

[9] F Mokhtarian, F Mohanna. Robust corner tracking for multimedia applications[C]// International Conference on Image Processing, Newyork, USA: IEEE, 2002, 3:945-948.

[10] X C He and N H C Yung. Corner detector based on global and local curvature properties, Optical Engineering, Optical Engineering, 47(5), 057008 (May 2008).

[11] Jinxia Dai, Xiaochun Chen, Naiqing Chu. RESEARCH ON THE EXTRACTION AND CLASSIFICATION OF THE CONCAVE POINT FROM FIBER IMAGE[C]. The 12th IEEE International Conference on Signal Processing, Hangzhou China,2014: 709-712. 